\title{
RECENT DEVELOPMENT OF STUDIES ON SCALE EFFECT
}

\author{
T.IDA \\ Kanagawa University \\ Yokohama, Japan \\ T. KUBOTA \\ Kanagawa University \\ Yokohama, Japan
}

\author{
J. KUROKAWA \\ Yokohama National University \\ Yokohama, Japan \\ H. TANAKA \\ Toshiba Corporation \\ Yokohama, Japan
}

\section{. Introduction}

n 1986, a sub-committee was organized in Japan Society of Mechanical Engineers JSME) to standardize a new scale effect formula by studying the outstanding problems f scale effect such as dependence of $V$ (loss distribution coefficient) on specific peed, roughness effect and so on. Then the sub-committee established a new erformance conversion method and published it as JSME S 008 in 1989 [1]. After $s$ publication, the second sub-committee has been organized to revise the conversion lethod by collecting the results of further study.

he most difficult problem in the study of scale effect is that the accuracy of the leasurement of prototype efficiency is incompetent to verify the scale effect. herefore, most of the study was conducted by using both theoretical analyses on umerical models of hydraulic machines and its verification by laboratory model tests. or this study, various numerical models which may simulate the performance of a hole machine were developed independently at institutes and industries in Japan. lost of them employed 3D flow analysis for major parts of the machines. In order to isess the wall friction, they used boundary layer calculation or wall friction loss llculation using flat plate analogy. These numerical models were refined and uproved by comparing their simulation results with performance characteristics tained by laboratory model tests. By using these models, the hydraulic loss in the achines were separated into scalable loss and non-scalable loss and the scale effect on e scalable loss was assessed quantitatively.

lis paper describes the summary of the results of these studies and the essential part the newly proposed performance conversion method which will be published soon as lew version of JSME S 008 . 


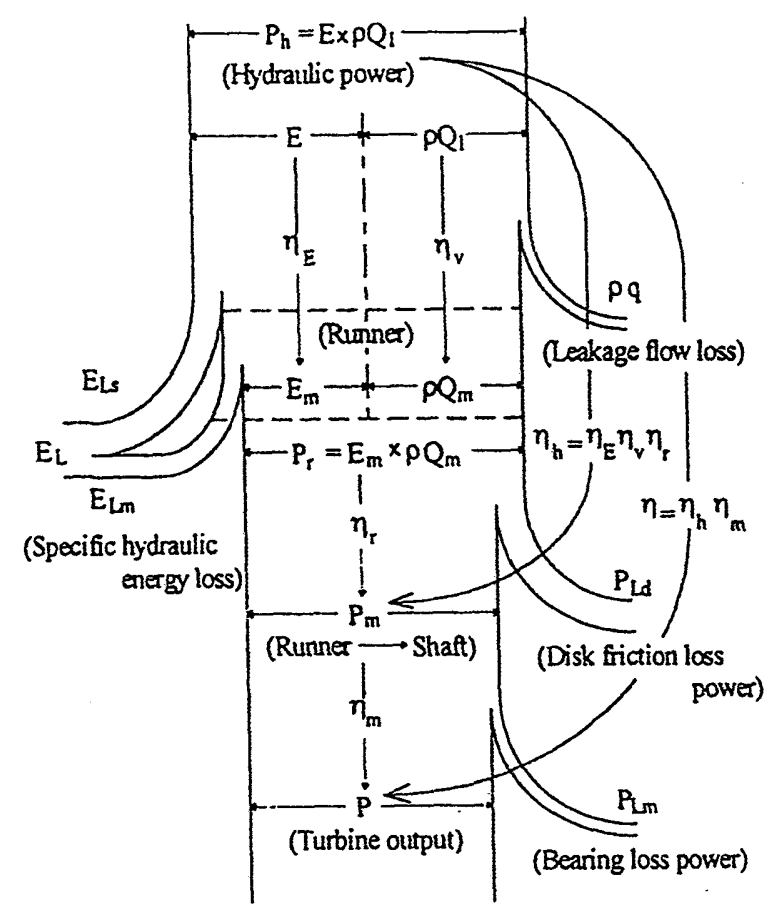

a) Turbine

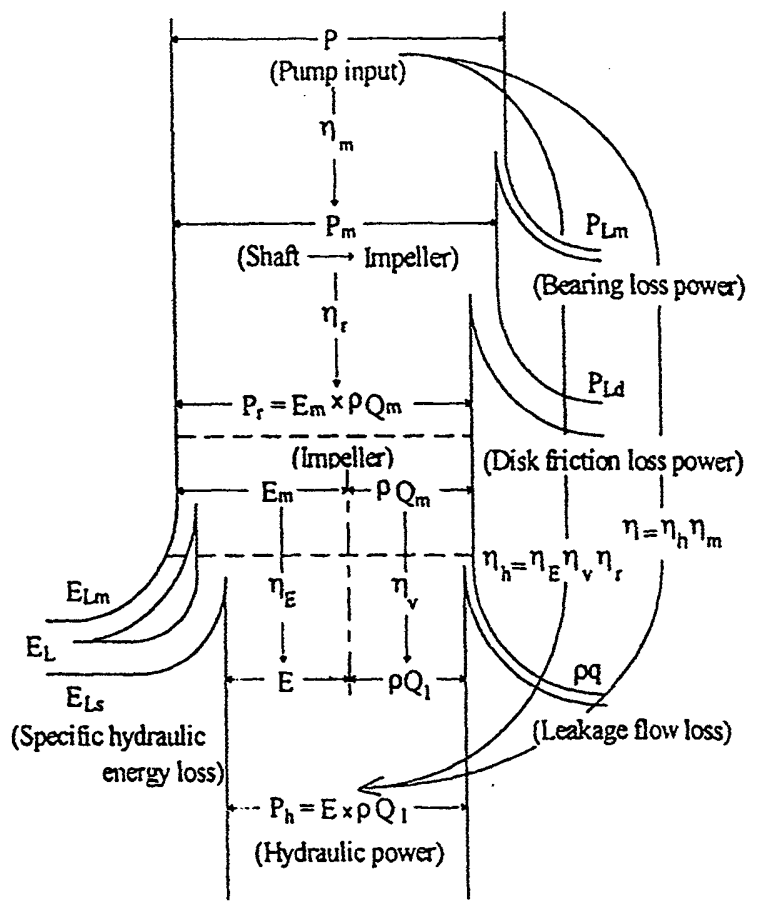

b) Pump

Fig. 1 Flux diagrams of hỵdraulic machines

\section{Structure of the New Scale Effect Formula}

The energy flux through hydraulic machines is conceptually represented by the diagrams set out in Fig. 1 a) and b). As indicated in these diagrams, the hydraulic efficiency of hydraulic machine $\eta_{h}$ is given by the product of the following three internal efficiencies.

$$
\eta_{\mathrm{h}}=\eta_{\mathrm{E}} \eta_{\mathrm{v}} \eta_{\mathrm{r}}
$$

where, $\eta_{E}$ is specific energy efficiency concerning all specific hydraulic energy loss in main water passages, $\eta_{v}$ is volumetric efficiency concerning leakage loss and $\eta_{r}$ is power efficiency of runner/impeller concerning disk friction.

Scale effect observed in performance characteristics of geometrically homologous hydraulic machines is caused by the change of wall friction loss due to the difference of Reynolds number and the relative roughness of the wall surface. Since the wall friction mostly causes dissipation of the specific hydraulic energy of the main flow, scale effect appears primarily in the specific hydraulic energy of the machine $E$. Scale effect also appears in leakage flow loss and in disk friction loss of the runner/impeller due to the difference of Reynolds number.

In the new performance conversion method, conversion factors for each internal efficiencies proposed by $T$. Ida are newly introduced. [5] They are specific energy efficiency scale factor $F_{E}=\eta_{E P} / \eta_{E M}$, volumetric efficiency scale factor $F_{\mathrm{v}}=\eta_{\mathrm{vP}} / \eta_{\mathrm{vM}}$ and power efficiency scale factor $F_{\mathrm{r}}=\eta_{\mathrm{rP}} / \eta_{\mathrm{rM}}$, where subscript $\mathrm{P}$ indicates the values of the prototype and $M$ for those of the model. By using these scale factors, the hydraulic efficiency of a model can be converted to the prototype 
efficiency as set out below.

$$
\eta_{\mathrm{hP}}=F_{\mathrm{E}} F_{\mathrm{v}} F_{\mathrm{r}} \eta_{\mathrm{hM}}
$$

These scale factors are standardized in the new conversion method as explained hereinafter.

Scale effect causes also shifting of performance. These scale factors are also applicable to the conversion of $E, Q$ and $P$, respectively. [2]

\section{Turbine}

$$
\begin{array}{rlrl}
E_{\mathrm{P}} & =F_{\mathrm{E}}^{-1}\left(\frac{n_{\mathrm{P}}}{n_{\mathrm{M}}}\right)^{2}\left(\frac{D_{\mathrm{P}}}{D_{\mathrm{M}}}\right)^{2} E_{\mathrm{M}} & E_{\mathrm{P}}=F_{\mathrm{E}}\left(\frac{n_{\mathrm{P}}}{n_{\mathrm{M}}}\right)^{2}\left(\frac{D_{\mathrm{P}}}{D_{\mathrm{M}}}\right)^{2} E_{\mathrm{M}} \\
Q_{\mathrm{P}}=F_{\mathrm{v}}^{-1}\left(\frac{n_{\mathrm{P}}}{n_{\mathrm{M}}}\right)\left(\frac{D_{\mathrm{P}}}{D_{\mathrm{M}}}\right)^{3} Q_{\mathrm{M}} & Q_{\mathrm{P}}=F_{\mathrm{v}}\left(\frac{n_{\mathrm{P}}}{n_{\mathrm{M}}}\right)\left(\frac{D_{\mathrm{P}}}{D_{\mathrm{M}}}\right)^{3} Q_{\mathrm{M}} \\
P_{\mathrm{P}}=F_{\mathrm{r}}\left(\frac{n_{\mathrm{P}}}{n_{\mathrm{M}}}\right)^{3}\left(\frac{D_{\mathrm{P}}}{D_{\mathrm{M}}}\right)^{5} P_{\mathrm{M}} & P_{\mathrm{P}}=F_{\mathrm{r}}^{-1}\left(\frac{n_{\mathrm{P}}}{n_{\mathrm{M}}}\right)^{3}\left(\frac{D_{\mathrm{P}}}{D_{\mathrm{M}}}\right)^{5} P_{\mathrm{M}}
\end{array}
$$

Pump

\section{New Formula for Conversion of Specific Energy Efficiency}

One of the major changes from the existing method to the new method is the use of relative scalable loss $\delta_{E}$ instead of loss distribution coefficient $V$. The definition of $\delta_{\mathrm{E}}$ is given by the following equation.

$$
\delta_{\mathrm{E}}=E_{\mathrm{Lf}} / E
$$

where, $E_{\mathrm{Lf}}$ is the scalable specific energy loss due to wall friction.

On the other hand, the loss distribution coefficient $V^{r}$ used in IEC Publication 995, which is denoted as $V_{\text {IEC }}$ or that in the existing version of JSME S 008, which is denoted as $V_{\text {ISME }}$, is defined as follows;

$$
\begin{gathered}
V_{\mathrm{IEC}}=\frac{(\text { Total scalable losses including disk friction, etc. })}{[(\text { Total scalable loss })+(\text { Total non-scalable loss })]} \\
V_{\mathrm{JSME}}=E_{\mathrm{Lf}} / E_{\mathrm{L}}=E_{\mathrm{Lf}} /\left(E_{\mathrm{Lf}}+E_{\mathrm{Lns}}\right)
\end{gathered}
$$

Whichever $V$ is used, the problem to use these $V$ values is that they include nonscalable loss $E_{\mathrm{Lns}}$ and others in their denominators which could be significantly affected by the difference of specific speed or individual design and, consequently, they could vary considerably from their standardized values depending on individual machine design. Also, it has been pointed out that, since these $V$ values were standardized for average machines, the prototype efficiency stepped up from poor efficiency model by using these values would be unreasonably too high.

It is expected that relative scalable loss $\delta_{\mathrm{E}}$ is less affected than $V$ by the difference of specific speed or individual design since it does not include non-scalable loss like $E_{\text {Lns }}$. The values of $\delta_{E}$ for Francis turbines and pump turbines, which are obtained 


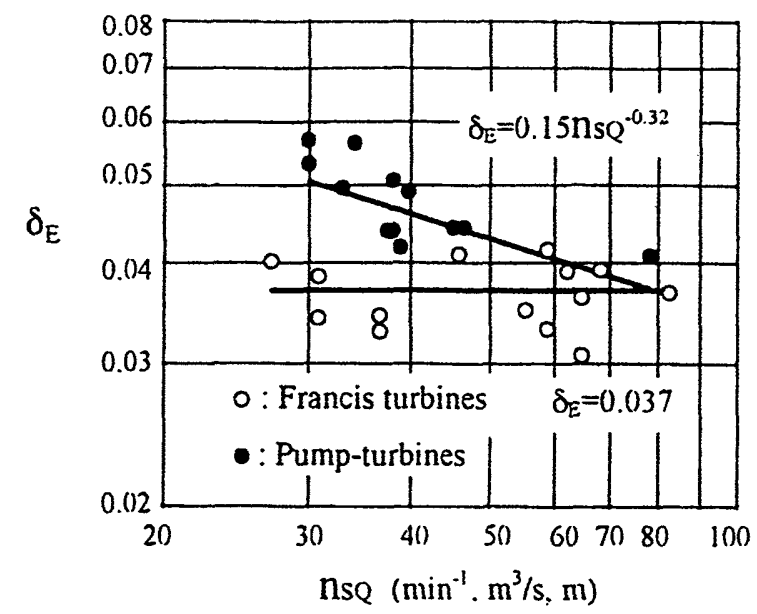

Fig. 2 Scalable loss $\delta_{E}$ versus specific speed

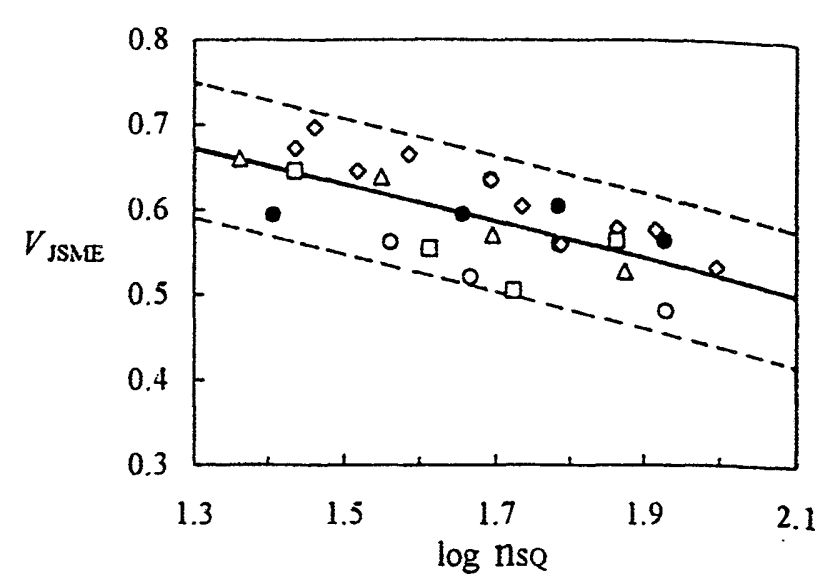

Fig. $3 V_{\text {JSME }}$ versus specific speed

from the investigation using various numerical models are shown in Fig. 2 . Apparently, their scattering is less than that of $V_{\mathrm{JSME}}$ [2], which is shown in Fig. 3, for example.

Hence, $\delta_{\mathrm{E}}$ is selected instead of $V$ as the standardized reference of the scalable loss. When the flow condition in both prototype and model is homologous, the ratio of their relative scalable loss denoted as $\Lambda$ is given as follows;

$$
\Lambda=\left(\delta_{\mathrm{EP}} / \delta_{\mathrm{EM}}\right)=\left(C_{\mathrm{fP}} / C_{\mathrm{fM}}\right)
$$

where, $C_{\mathrm{f}}$ is the friction loss coefficient for main water passages.

Then new scale effect formulae for the conversion of the specific energy efficiency are proposed by T. Ida.[3] They are written as follows;

$$
\text { For turbine: } \quad F_{\mathrm{E}}=\frac{1}{1-(1-\Lambda) \delta_{\mathrm{E}}} \quad \text { or } \quad \Delta \eta_{\mathrm{E}}=\eta_{\mathrm{EM}}\left[\frac{1}{1-(1-\Lambda) \delta_{\mathrm{E}}}-1\right]
$$

For pump: $\quad F_{E}=1+(1-\Lambda) \delta_{E} \quad$ or $\quad \Delta \eta_{E}=\eta_{E M}(1-\Lambda) \delta_{E}$

For simplification, all the model values in this paper are assumed to be those measured at the reference Reynolds number.

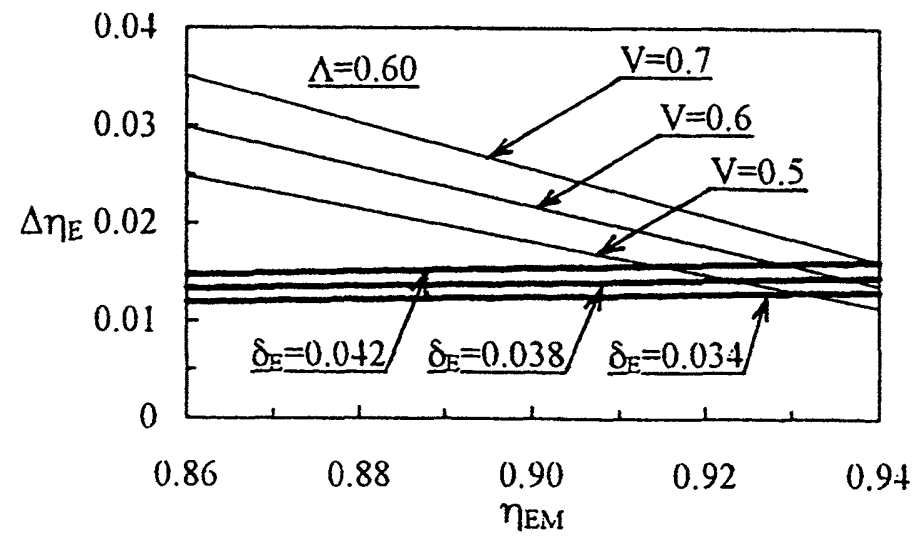

Fig. 4 Efficiency step up $\Delta \eta_{E}$ versus model efficiency $\eta_{E M}$ 
The values of $\delta_{E}$ and $V$, which were collected from the results of the numerical model analysis, show some scattering as shown in Figs. 2 and 3. The uncertainty of $\Delta \eta_{\mathrm{E}}$ caused by such scattering is examined and shown in Fig. 4 . It is observed that the scattering of $\Delta \eta_{E}$ caused by the scattering of $\delta_{E}$ is less than that caused by $V$. Thus, by using the new formulae based on $\delta_{E}$, the accuracy of $\Delta \eta_{E}$ can be improved considerably and the unreasonably large efficiency step up for poor efficiency turbines can be avoided.

\section{Friction Coefficient Ratio Considering Roughness Effect}

The effects of surface roughness on the wall friction loss are studied by $T$. Ida using the analogy of the friction loss of flat plate. Basic equations of the fricion loss coefficient for flat plate which are used as a basis for the discussions hereinafter are shown below.

For smooth surface: $C_{\mathrm{fS}}=0.455\left(\log _{10} R e_{\mathrm{L}}\right)^{-2.58}$

For rough surface : $C_{\mathrm{fR}}=\left[1.89-1.62 \log _{10}\left(k_{\mathrm{S}} / L\right)\right]^{-2.5}$ where, $k_{\mathrm{S}}$ is the equivalent sand roughness and $L$ is the length of the plate.

The admissible sand roughness can be obtained as the solution of $C_{\mathrm{tS}}=C_{\mathrm{fR}}$. The result is;

$$
\left(k_{\text {Sadm }} / L\right)=10{ }^{\left\{(1.89 / 1.62)-(1 / 1.62)\left\{\left(\log _{10} R e\right)^{2.58} / 0.455\right]^{0.4}\right\}} \approx 24.0 R e_{L}^{-0.931}
$$

The extreme right is the approximation for the middle equation. The admissible roughness thus obtained is shown in Fig. 5. The admissible roughness given by the Schlichting's equation is also plotted for comparison.

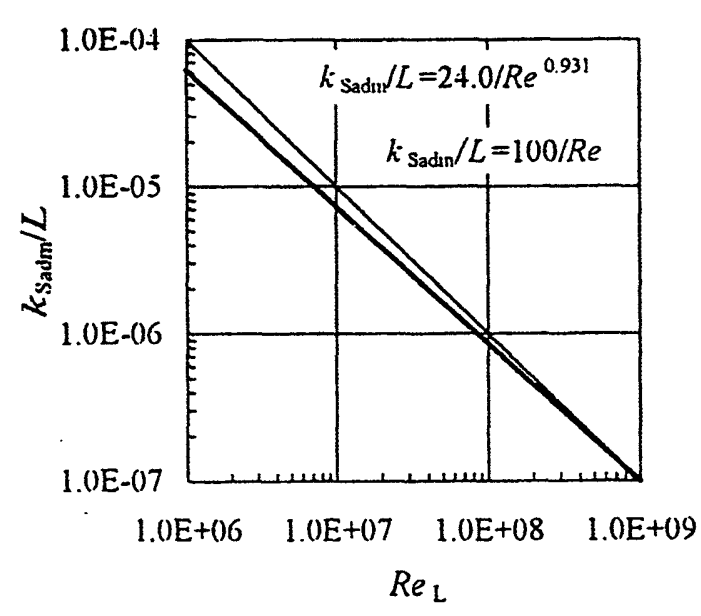

Fig. 5 Admissible sand roughness versus Reynolds number

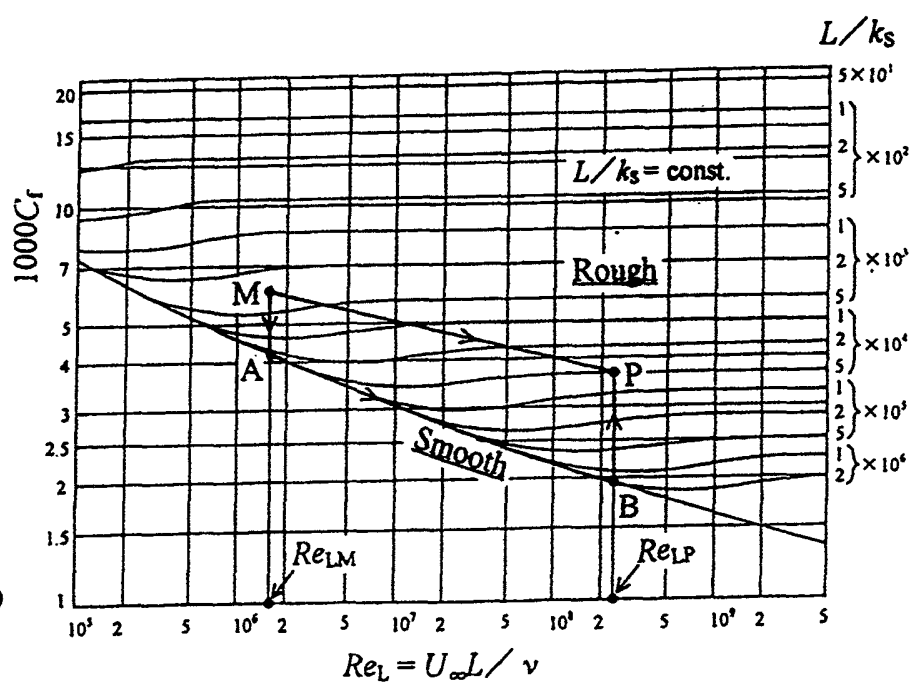

Fig. 6 Friction loss coefficient of sand roughened flat plate 
In general, actual surface roughness $e$, which is usually expressed in terms of $R a$, is not the same as $k_{\mathrm{S}}$. Therefore, when using the admissible sand roughness obtained above, the following conversion is required.

$$
e_{\text {adm }}=k_{\text {Sadm }} / C_{\mathrm{k}}
$$

where, $C_{\mathrm{k}}$ is a coefficent to be obtained experimentally. Unfortunately, although a few results of the experimental studies on $C_{\mathrm{k}}$ were published, they did not sufficiently coincide with each other. Further study is required in this regard.

The variation of friction loss coefficient $C_{\mathrm{f}}$ for different Reynolds number and surface roughness is shown in Fig. 6 . In this diagram, point $\mathrm{M}$ represents $C_{\mathrm{f}}$ for a rough model (strictly speaking, a model not perfectly smooth) and point $P$ for a rough prototype (similarly, a prototype not perfectly smooth). Here, we consider a smooth model $A$ which has the same Reynolds number with $M$ and a smooth prototype $B$ with the same Reynolds number with $P$. Then, the conversion of the friction loss coefficient from the rough model $M$ to the rough prototype $P$ is divided into three steps; namely, $M \rightarrow A \rightarrow B \rightarrow P$. Thus, the ratio of friction loss coefficient, $\Lambda_{M \rightarrow P}$, may be expressed by the following equation.

$$
\Lambda_{M \rightarrow P}=\Lambda_{M \rightarrow A} \times \Lambda_{A \rightarrow B} \times \Lambda_{B \rightarrow P}=\Lambda_{A \rightarrow B} \times \Lambda_{B \rightarrow P} / \Lambda_{A \rightarrow M}
$$

Firstly, by Eq. (11), $\Lambda_{A \rightarrow B}$ (smooth model to smooth prototype) is given as the first line below and it can be approximated with good accuracy by the equation at the second line.

$$
\begin{aligned}
\Lambda_{\mathrm{A} \rightarrow \mathrm{B}} & =\left(C_{\mathrm{fSB}} / C_{\mathrm{fSA}}\right)=\left[\left(\log _{10} R e_{\mathrm{LP}}\right) /\left(\log _{10} R e_{\mathrm{LM}}\right)\right]^{-2.58} \\
& \approx\left(R e_{\mathrm{LP}} / R e_{\mathrm{LM}}\right)^{-0.155}
\end{aligned}
$$

Secondly, $\Lambda_{\mathrm{B} \rightarrow \mathrm{P}}$ (smooth machine to rough machine) is obtained. By combining Eqs. (11) and (12), it is given as the first line below and it can be closely approximated by the equation at the second line.

$$
\begin{aligned}
\Lambda_{\mathrm{B} \rightarrow \mathrm{P}} & =\left(C_{\mathrm{fRP}} / C_{\mathrm{fSB}}\right)=\left[1.89-1.62 \log _{10}\left(k_{\mathrm{SP}} / L_{\mathrm{P}}\right)\right]^{-2.5} / 0.455\left(\log _{10} R e_{\mathrm{LP}}\right)^{-2.58} \\
& \approx 0.678\left(k_{\mathrm{SP}} / L_{\mathrm{P}}\right)^{0.18} \operatorname{Re}_{\mathrm{LP}}{ }^{0.155}
\end{aligned}
$$

Similarly $\Lambda_{\mathrm{A} \rightarrow \mathrm{M}}$ is obtained as follows;

$$
\Lambda_{\mathrm{A} \rightarrow \mathrm{M}}=0.678\left(k_{\mathrm{SM}} / L_{\mathrm{M}}\right)^{0.18} \operatorname{Re}_{\mathrm{LM}}{ }^{0.155}
$$

By substituting Eqs. (16), (17) and (18) into Eq. (15), we obtain;

$$
\begin{aligned}
\Lambda_{\mathrm{M} \rightarrow \mathrm{P}} & =\left(R e_{\mathrm{LP}} / R e_{\mathrm{LM}}\right)^{-0.155}\left[\left(k_{\mathrm{SP}} / L_{\mathrm{P}}\right) /\left(k_{\mathrm{SM}} / L_{\mathrm{M}}\right)\right]^{0.18}\left(R e_{\mathrm{LP}} / R e_{\mathrm{LM}}\right)^{0.155} \\
& =\left[\left(k_{\mathrm{SP}} / L_{\mathrm{P}}\right) /\left(k_{\mathrm{SM}} / L_{\mathrm{M}}\right)\right]^{0.18}
\end{aligned}
$$

In the above equation, $L$ represents the length of vanes or passages. Therefore, in case of homologous machines, $\left(L_{\mathrm{P}} / L_{\mathrm{M}}\right)=\left(D_{\mathrm{P}} / D_{\mathrm{M}}\right)$. Also, using actual roughness $e,\left(k_{\mathrm{SP}} / k_{\mathrm{SM}}\right)$ can be substituted by $\left(e_{\mathrm{P}} / e_{\mathrm{M}}\right)$. After all, $\Lambda_{\mathrm{M} \rightarrow \mathrm{P}}$ is obtained as follows; 


$$
\Lambda_{\mathrm{M} \rightarrow \mathrm{P}}=\left(D_{\mathrm{P}} \cdot D_{\mathrm{M}}\right)^{-0.18}\left(e_{\mathrm{P}} / e_{\mathrm{M}}\right)^{0.18}
$$

If the surface roughness of the model is smooth i.e. $e_{\mathrm{M}} \leq e_{\mathrm{adm} M}$, the following equation can be used for the conversion from a smooth model to a rough prototype.

$$
\Lambda_{\mathrm{M} \rightarrow \mathrm{P}}=\left(D_{\mathrm{P}} / D_{\mathrm{M}}\right)^{-0.18}\left(e_{\mathrm{P}} / e_{\mathrm{admM}}\right)^{0.18}
$$

Since a prototype has generally larger Reynolds number and smaller admissible roughness, its surface roughness is mostly hydraulically rough. Especially, surfaces of runner/impeller and distributor are exposed to high velocity flow and subjected to significant roughness effect. Therefore, it is generally necessary to apply Eq. (20) or (21) to the wall friction loss on these areas taking into account the difference of the roughness of both model and prototype; namely, $\left(e_{\mathrm{P}} / e_{\mathrm{M}}\right) \neq 1$. Regarding the other areas, most of them are painted and their roughness is almost identical for both model and prototype. Therefore, for these areas, Eq. (20) or (21) assuming $\left(e_{\mathrm{P}} / e_{\mathrm{M}}\right)=1$ can be applied.

Then. similar to the existing code JSME S 008, a coefficient $\beta$ which is defined by the following equation is introduced.

$$
\beta=\frac{\text { (Friction loss in runner / impeller and distributor) }}{\text { (Total friction loss in main water passage) }}
$$

By using $\beta$, the friction loss coefficient ratio $\Lambda_{M \rightarrow P}$ applicable to actual machine is obtained as follows;

$$
\begin{aligned}
\Lambda_{\mathrm{M} \rightarrow \mathrm{P}} & =(1-\beta)\left(D_{\mathrm{P}} / D_{\mathrm{M}}\right)^{-0.18}(1 / 1)^{0.18}+\beta\left(D_{\mathrm{P}} / D_{\mathrm{M}}\right)^{-0.18}\left(e_{\mathrm{P}} / e_{\mathrm{M}}\right)^{0.18} \\
& =\left(D_{\mathrm{P}} / D_{\mathrm{M}}\right)^{-0.18}\left[(1-\beta)+\beta\left(e_{\mathrm{P}} / e_{\mathrm{M}}\right)^{0.18}\right]
\end{aligned}
$$

The value of $\beta$ can be estimated by the simulation analysis using numerical models. Some examples of its value at optimum condition are shown in Fig. 7. It becomes larger for higher specific speed machines, however, mostly it remains within the range of $0.6 \sim 0.85$.

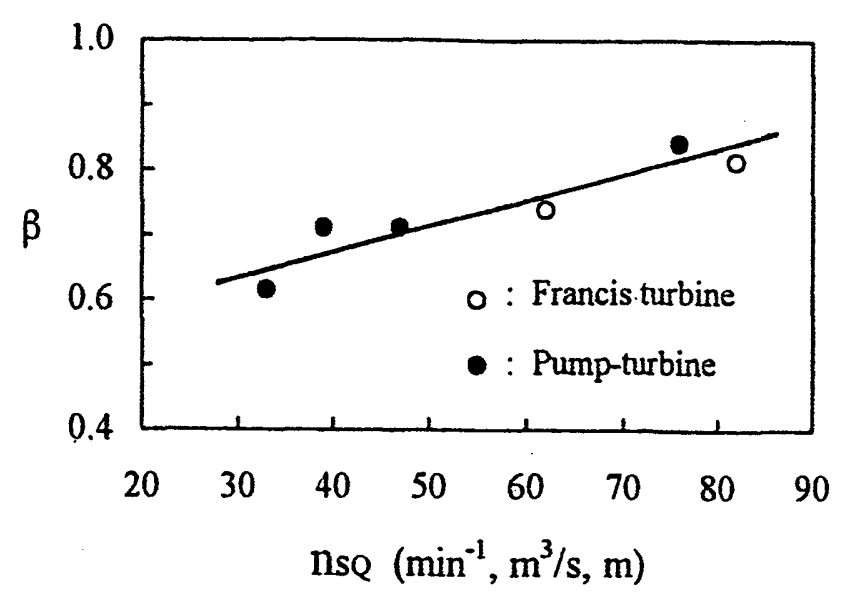

Fig. $7 \beta$ versus specific speed 


\section{Influence of the Approximation of Principal Coefficients}

\subsection{INFLUENCE OF RELATIVE SCALABLE LOSS $\delta_{\mathrm{E}}$}

As shown in Fig. 2, the scattering of $\delta_{E}$ ranges over $0.037 \pm 0.004$. The result of an example to examine the influence of this scattering is shown in Fig. 8. As observed in this diagram, the difference of $\Delta \eta_{E}$ caused by the above scattering of $\delta_{E}$ is only $\pm 0.13 \%$. It suggests that a single value of $\delta_{\mathrm{E}}=0.037$ can be used practically as a standardized value for all Francis turbines of various specific speed.

\subsection{INFLUENCE OF DEPENDENCE COEFFICIENT OF ROUGHNESS RATIO $\beta$}

As shown in Fig. 7, the value of $\beta$ varies over a range of 0.60 to 0.85 . Another examination to see the influence of the different values of $\beta$ was conducted. An example of the results of the conversion using different values of $\beta$ is shown in Fig. 9. It indicates that, if $\beta$ is selected within a range of $0.8 \pm 0.2$, the dispersion of the resultant prototype efficiency does not exceed $\pm 0.1 \%$. Thus the influence of the deviation of $\beta$ on $\Delta \eta_{E}$ is small. It suggests that a constant value $\beta=0.7$ may be used for all Francis turbines and pump-turbines in practical application.

In this figure, $K_{\mathrm{m}}$ and $K_{\mathrm{p}}$ are the dimensionless flow coefficient and output power coefficient, respectively, which are defined as follows:

$$
\begin{aligned}
& K_{\mathrm{m}}=\left(Q_{1} / A_{\mathrm{R} 1}\right) /(2 E)^{1: 2} \\
& K_{\mathrm{p}}=P /\left[\rho A_{\mathrm{R} 1}(2 E)^{1 / 2} E\right]
\end{aligned}
$$

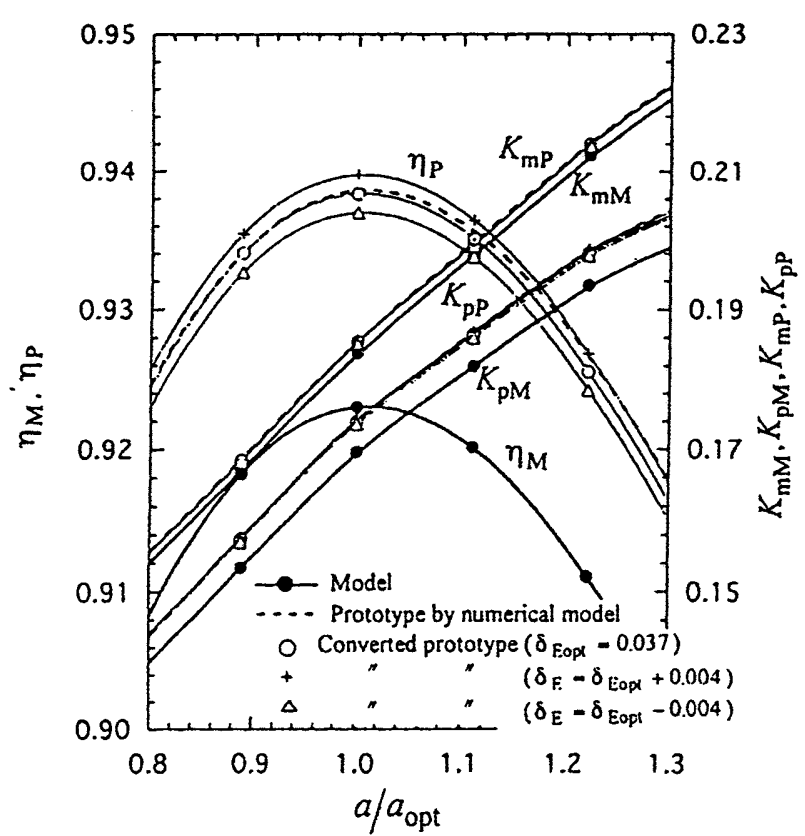

Fig. 8 Influence of the difference of $\delta_{E}$ on the converted prototype performances (Francis turbine: $\left.\mathrm{n}_{\mathrm{SQ}}=62,\left(e_{\mathrm{P}} / e_{\mathrm{M}}\right)=4.0\right)$

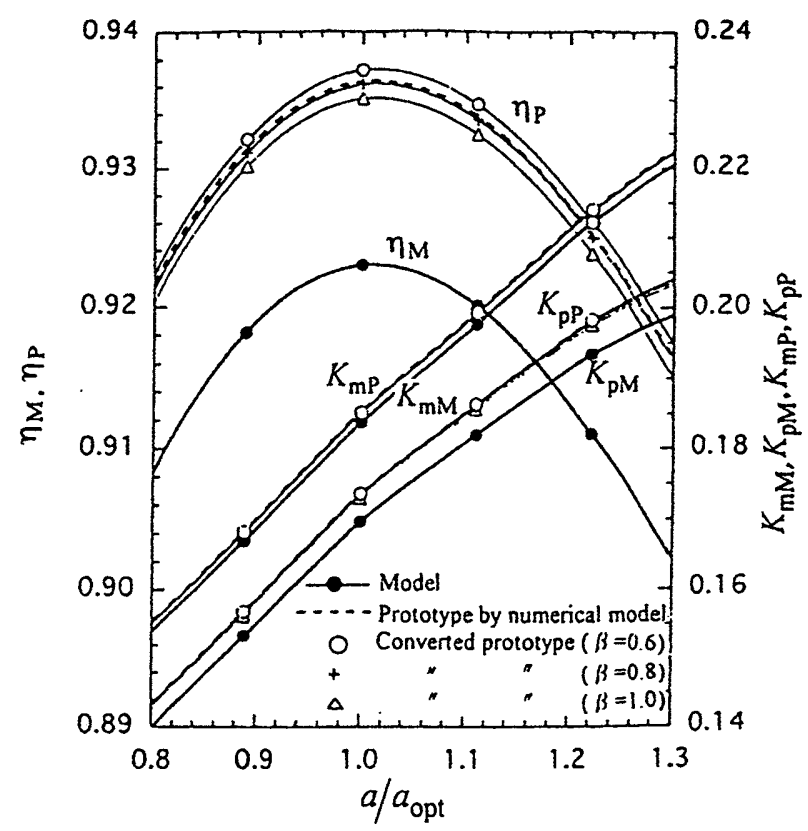

Fig. 9 Influence of the difference of $\beta$ on the converted prototype performances (Francis turbine: $\mathrm{n}_{\mathrm{sQ}}=62,\left(e_{\mathrm{p}} / e_{\mathrm{M}}\right)=4.0$ ) 
It should be noted that $K_{\mathrm{m}}$ and $K_{\mathrm{p}}$ of the prototype are significantly influenced by scale effect since they include $E$, which is most likely to be affected by scale effect, in their denominators.

\section{Conversion of Volumetric Efficiency}

Volumetric efficiency $\eta_{\mathrm{v}}$ is determined by leakage flow loss and it is subjected to scale effect. In the new conversion method, it is converted to the prototype value by the following equation.

$$
\eta_{\mathrm{vP}}=F_{\mathrm{v}} \eta_{\mathrm{vM}}
$$

The value of $\eta_{v M}$ obtained by the numerical analysis is plotted against specific speed and shown in Fig. 10. Those for Francis turbines and pump-turbines are represented by a common single curve. [4]

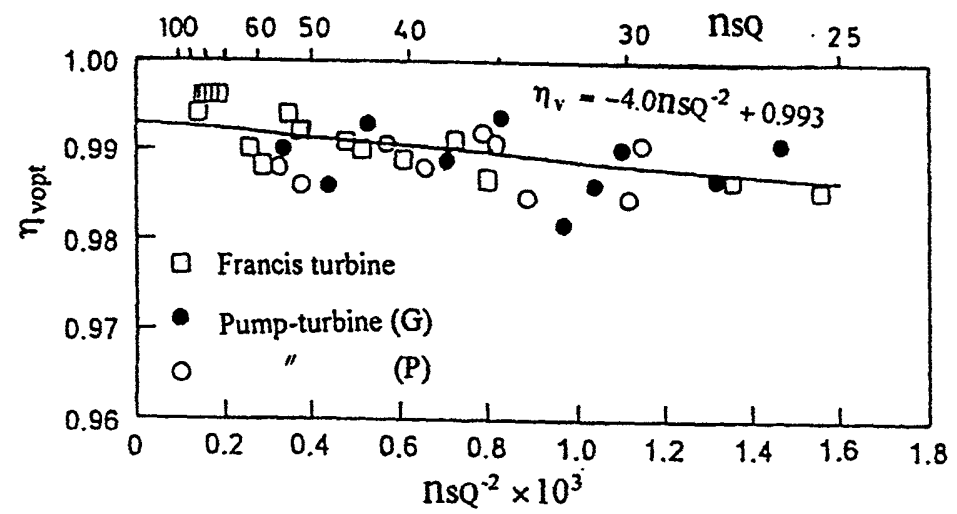

Fig. 10 Volumetric efficiency $\eta_{\mathrm{vM}}$ versus specific speed

Detailed study to determine $\Lambda_{\mathrm{v}}$ and $F_{\mathrm{v}}$ is conducted by $\mathrm{T}$. Ida. [5] The scale factor $F_{\mathrm{v}}$ can be expressed as follows;

$$
\begin{array}{ll}
F_{\mathrm{v}}=1 /\left[\eta_{\mathrm{vM}}+\Lambda_{\mathrm{v}}\left(1-\eta_{\mathrm{vM}}\right)\right] & \text { (for turbine) } \\
F_{\mathrm{v}}=\left[\left(1-\Lambda_{\mathrm{v}}\right) / \eta_{\mathrm{vM}}\right]+\Lambda_{\mathrm{v}} & \text { (for pump) }
\end{array}
$$

As a result of the study, it is made clear that $F_{\mathrm{v}}$ may be regarded as $0.997 \sim 0.998$ within the practical range of $\left(R e_{\mathrm{P}} / R e_{\mathrm{M}}\right)=5 \sim 25$ and $\mathrm{n}_{\mathrm{SQ}}=25 \sim 100$, when the geometry of the seal clearances of both model and prototype is homologous.

\section{Conversion of Power Efficiency of Runner/impeller}

Similar to volumetric efficiency, power efficiency $\eta_{\mathrm{r}}$ concerning disk friction of runner/impeller can be converted by the following formula.

$$
\eta_{\mathrm{rP}}=F_{\mathrm{r}} \eta_{\mathrm{rM}}
$$

The value of $\eta_{T M}$ is investigated by using the numerical models and the results are shown in Fig. 11. 


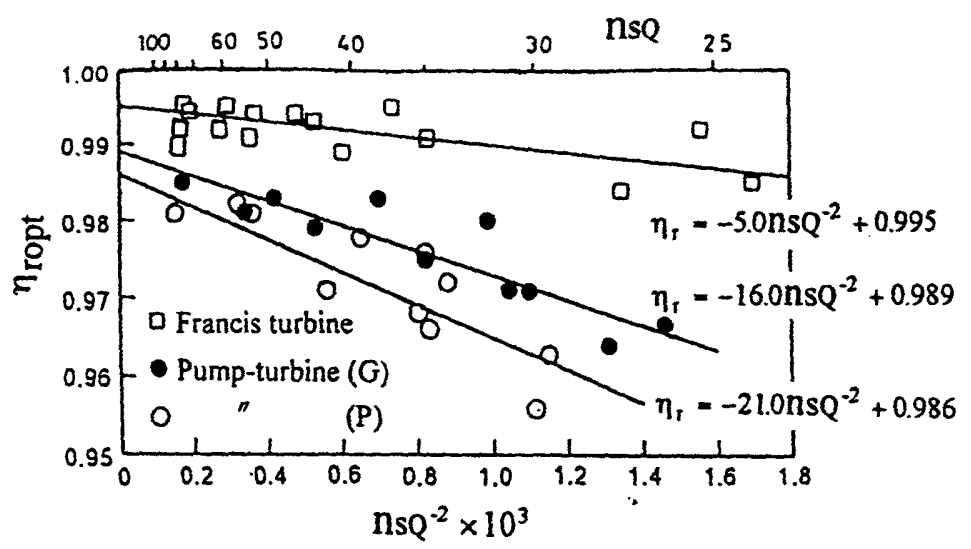

Fig. 11 Power efficiency of runner/impeller $\eta_{r M}$ versus specific speed

The scale factor $F_{\mathrm{r}}$ is given by the following equation. [5]

$$
\begin{array}{ll}
F_{\mathrm{r}}=\left[\left(1-\Lambda_{\mathrm{r}}\right) / \eta_{\mathrm{rM}}\right]+\Lambda_{\mathrm{r}} & \text { (for turbine) } \\
F_{\mathrm{r}}=1 /\left[\left(1-\Lambda_{\mathrm{r}}\right) \eta_{\mathrm{rM}}+\Lambda_{\mathrm{r}}\right] & \text { (for pump) }
\end{array}
$$

where, $\Lambda_{r}$ is the ratio of the disk friction loss coefficient. If it is assumed that the surface roughness relevant to the disk friction is hydraulically rough, the following approximate equation is obtained.

$$
\Lambda_{\mathrm{r}}=\left(D_{\mathrm{P}} / D_{\mathrm{M}}\right)^{-0.27}\left(e_{\mathrm{P}} / e_{\mathrm{M}}\right)^{0.12}
$$

Finally, the following equations for $F_{\mathrm{r}}$ are derived.

$$
\begin{array}{ll}
F_{\mathrm{r}}=\left(0.388 / \eta_{\mathrm{rM}}\right)+0.612 & \text { (for turbine) } \\
F_{\mathrm{r}}=1 /\left(0.995-8.2 \mathrm{n}_{\mathrm{sQ}}{ }^{-2}\right) & \text { (for pump) }
\end{array}
$$

\section{Conclusion}

New performance conversion method with regard to scale effect is proposed for the new version of JSME S 008. Compared with the existing version, it is expected that the new method gives more reasonable and accurate conversion. In addition, introduction of scale factors makes the calculation of performance shifting due to scale effect much easier.

\section{Reference}

1 JSME S 008 (1989) Performance conversion method for hydraulic turbines and pumps

2 Ida, T. et al. (1990) Advanced scale-up formulae for performances of hydro-turbines and pump-turbines considering surface roughness, Proc. IAHR Symp. in Belgrade 1, B4

3 Ida, T. (1994) New formula for efficiency step-up of hydraulic turbines, Proc. IAHR Symp. in Beijing 2, 827840

4 Kurokawa, J. et al. (1994) Accurate determination of volumetric and mechanical efficiencies and leakage behaviour of Francis turbine and Francis pump-turbine, Proc. IAHR SYMP. in Beijing 2, 889-900

5 Ida, T. (1995) New formulae for scaling-up hydraulic efficiency of hydraulic turbines, Journal of Hydraulic Research 33, 2, 147-162 